\title{
Evidence-based guideline implementation in low and middle income countries: lessons for mental health care
}

\author{
Mary Docherty ${ }^{1}$, Kate Shaw ${ }^{2}$, Lucy Goulding ${ }^{2}$, Hannah Parke², Erica Eassom² ${ }^{2}$ Farnoosh Ali ${ }^{2}$ \\ and Graham Thornicroft ${ }^{3 *}$
}

\begin{abstract}
Background: There is a significant treatment gap in provision of effective treatment for people with mental disorders globally. In some Low and Middle Income Countries (LMICs) this gap is $90 \%$ or more in terms of untreated cases. Clinical practice guidelines (CPGs) are one tool to improve health care provision. The aim of this review is to examine studies of the effectiveness of evidence-based CPG implementation across physical and mental health care, to inform mental healthcare provision in low and middle income countries (LMICs), and to identify transferable lessons from other non-communicable diseases to mental health.
\end{abstract}

Methods: A systematic literature review employing narrative synthesis and utilising the tools developed by the Cochrane Effective Practice and Organisation of Care (EPOC) group was conducted. Experimental studies of CPG implementation relating to non-communicable diseases, including mental disorders, in LMICs were retrieved and synthesised.

Results: Few (six) studies were identified. Four cluster randomised controlled trials (RCTs) related to the introduction of CPGs for non-communicable diseases in physical health; one cluster-RCT included CPGs for both a noncommunicable disease in physical health and mental health, and one uncontrolled before and after study described the introduction of a CPG for mental health. All of the included studies adopted multi-faceted CPG implementation strategies and used education as part of this strategy. Components of the multi-faceted strategies were sometimes poorly described. Results of the studies included generally show statistically significant improvement on some, but not all, outcomes.

Conclusion: Evidence for the effectiveness of interventions to improve uptake of, and compliance with, evidencebased CPGs in LMICs for mental disorders and for other non-communicable diseases is at present limited. The sparse literature does, however, suggest that multifaceted CPG implementation strategies that involve an educational component may be an effective way of improving guideline adherence and therefore of improving clinical outcomes. Further work is needed to examine cost-effectiveness of CPG implementation strategies in LMICs and to draw conclusions on the transferability of implementation experience in physical health care to mental health practice settings. Strategies to ensure that CPGs are developed with clear guidance for implementation, and with explicit, methods to evaluate them should be a priority for mental health researchers and for international agencies.

Keywords: Guideline implementation, Clinical practice guideline, Evidence-based practice, Implementation science, Implementation strategies, Low income countries, Middle income countries, Mental health, Physical health, Systematic literature review

\footnotetext{
*Correspondence: graham.thornicroft@kcl.ac.uk

${ }^{3}$ Centre for Global Mental Health and Centre for Implementation Science,

Institute of Psychiatry, Psychology and Neuroscience, King's College

London, London, UK

Full list of author information is available at the end of the article
} 


\section{Background}

The global burden of mental, neurological and substance use (MNS) disorders is relentlessly high, resulting in long-term disability combined with premature mortality $[1,2]$. Untreated MNS disorders also have a negative impact on global health priorities [3], and may be associated with human rights abuses [4]. The majority of people with MNS disorders in LMICs are unable to access effective mental health care, with the treatment gap higher than $90 \%$ in many such countries [5-7]. The most recent estimates of the global burden of mental and neurological disorders suggest that these may be considerably greater than previously thought [8].

Issues related to quality improvement and implementation science are central to these challenges $[9,10]$. Health system constraints are recognised to be potent threats to the scale-up of access to evidence-based mental health care for people affected by MNS disorders in low- and middle-income countries (LMICs) [11]. Policy makers and planners play a critical role in the successful strengthening of mental health systems, but may not be appropriately equipped for the task. For example, in a mixed-methods study of the challenges faced during implementation of national mental health policy in South Africa, the key barriers included the low priority given to mental health care by planners, provincial bureaucracy around service coordination, insufficient staff for policymaking and service planning, and disinclination by some local authorities to lead mental health policy implementation [12].

In a qualitative study involving national and regional stakeholders in Ghana, South Africa, Uganda and Zambia, low perceived legitimacy of the problem of scaling up mental health services and inadequate government support were identified as factors perpetuating the low priority accorded to mental health care [13]. A survey of leaders and specialists in international mental health specifically identified a need for a more over-arching public health perspective among mental health policy-makers [14]. The lack of training and experience of clinicians to fulfil leadership roles in policy making and planning was particularly emphasised.

There is international consensus on the need for mental health system strengthening and for a specific focus on building the capacity of key stakeholders, including policy makers and planners and service users [15-18].

These issues speak to the quantity of mental health care available in LMICs. Indeed, the key mental health focus of the WHO is the mental health gap, namely the difference between true prevalence rates of mental disorders and treated prevalence rates. Such treated prevalence rates are sometimes expressed as treatment coverage. Nevertheless the usual way in which coverage is conceptualised and measured refers to contact coverage, i.e. the occurrence of any treatment encounter, whether or not this confers benefit to the patient. A more satisfactory definition refers to quality of care as expressed in terms of effectiveness coverage, which means the proportion of people with mental disorders, at a time point or over a time period, who receive effective treatment and care (see Fig. 1) [19].

Regarding implementation, it is clear in terms of the provision of mental health care that there are two central problems: weak or absent (1) national mental health plans, and (2) mechanisms to implement these national plans, including patient and practitioner level interventions such as evidence-based clinical practice guidelines, which are simply not put into practice. One can therefore speak of an implementation gap, which is a complex set of barriers standing in the way of better mental health care across most countries of the world [20]. Among the reasons for non-implementation of carefully constructed national level strategic plans, and local level treatment guidelines is the paucity of leadership skills for general health systems strengthening [17, 21-23].

Turning now to the quality of care, clinical practice guidelines (CPGs) are an established tool to support reduction in variation, improvement in quality and efficiency, and delivery of evidence-based care [24]. Over the last two decades there has been a relatively large production of mental health guidelines in high-income countries, particularly in the UK, Canada, New Zealand and Australia. With respect to these efforts however, only a small corollary body of research focused on their implementation [25]. Recognition of this knowledge gap and lack of clarity as to the benefits of implementing specific psychiatric guidelines in routine primary care or mental health specialist teams [26] has led to calls for more formal evaluations in this field [27].

A recent systematic review concluded that there was sufficient evidence from high income countries to view CPGs in mental healthcare as an essential asset if appropriately developed and implemented [28]. They showed trends towards improvement in process and patient outcomes following guideline implementation [28]. This supports the legitimacy of CPG development and implementation in LMICs as one of a number of possible tools to approach scale up of mental health care in these settings. On-going difficulties in methodology to evaluate implementation techniques has however been identified as a significant barrier to optimising the potential value of evidence-based guidelines as tools for population level improvements in care [28].

The context for this paper is therefore an appreciation that little has so far been published within the mental health field about implementation of evidence-based 


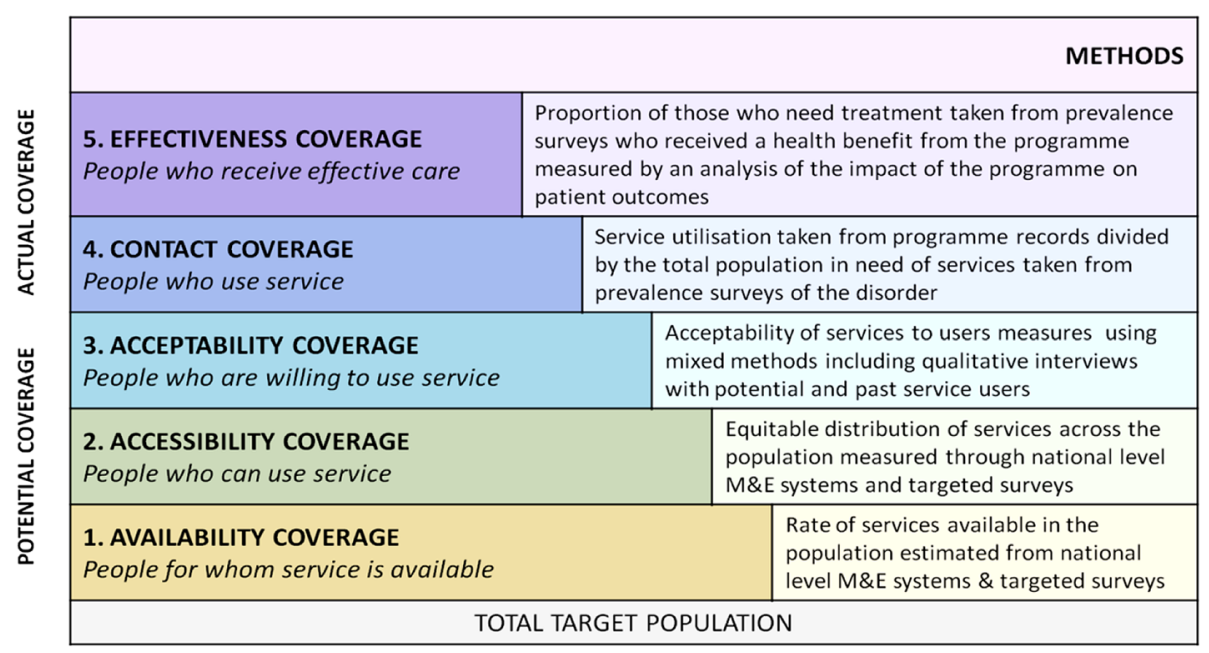

Fig. 1 Schema to show different types of treatment coverage [19, 24]

policies and practices in LMICs [29-31]. At the same time we wish to learn from other health sectors of how such progress in LMICs can be made. The aims of this paper are therefore to: (1) review evidence for CPG implementation in physical and mental health care settings in LMICs; (2) consider the transferability of lessons learned from implementation of CPGs in physical health to mental health in LMICs.

\section{Methods}

\section{Design of the review}

The systematic review was conducted following guidelines produced by the Cochrane Collaboration [32]. The PRISMA checklist was used to inform reporting of the review [33].

\section{Searches}

The following electronic databases were searched: MEDLINE, EMBASE, PsychInfo, Global Health and LILACS (Latin American and Caribbean Health Sciences Literature). The search strategy was prepared for MEDLINE (see Additional file 1: Web Appendix) and then translated to other databases following their requirements. Literature published since the earliest date indexed in each database up to the search date (October 2014) was retrieved. Duplicates were removed prior to screening.

Reference list checks of studies found during the electronic search were made. General searches were conducted using internet search engines and key authors in the field were asked to indicate potentially relevant studies. The review team did not have any funds to provide translation; however, Google Translate was used to translate the full text of three studies that were not reported in English. None of these papers met the inclusion criteria.

\section{Study inclusion and exclusion criteria \\ Design}

Studies relating to a non-communicable physical health condition using any of the following designs were included: randomised controlled trials (RCT), clusterRCTs, controlled clinical trials (CCT), controlled-beforeand-after studies (CBA) and interrupted time series (ITS) studies with concurrent controls.

Studies relating to a mental health condition using any of the following designs were included: randomised controlled trials (RCT), cluster-RCTs, controlled clinical trials $(\mathrm{CCT})$, controlled-before-and-after studies (CBA), interrupted time series (ITS) studies with concurrent controls, and uncontrolled before and after studies.

Included studies were permitted to have any number of sites. Studies with non-experimental designs were excluded. However, findings from process evaluation or qualitative studies conducted alongside experimental studies were included in the data extraction and synthesis of results.

\section{Countries and populations included}

Included studies were conducted in a low or middle income country. The World Bank Atlas method for 2014 defined low-income economies as those with a GNI per capita of \$1045 or less; middle-income economies had a GNI per capita of more than $\$ 1045$ but less than $\$ 12,746$. Studies conducted in high-income countries were excluded.

Eligible participants in included studies were children or adults of any age with a non-communicable disease-including mental and physical health conditions. Examples include: depression, dementia, coronary heart disease, cancer. Studies solely involving participants with 
communicable diseases (e.g. malaria, HIV, influenza) were excluded. Studies involving outcomes related to surgical site infection and hand hygiene were also excluded. Studies that included participants with both non-communicable and communicable diseases were included with outcomes for people with non-communicable diseases being the focus during synthesis and reporting of the results.

\section{Interventions}

Included studies described the introduction of a CPG relating to management of people who have a noncommunicable disease (including mental and physical health). The Institute of Medicine's definition of a CPG was adopted. This states that "clinical practice guidelines are statements that include recommendations intended to optimize patient care that are informed by a systematic review of evidence and an assessment of the benefit and harms of alternative care options" [34].

Studies that assessed the implementation of more than one CPG were included if at least one of the guidelines was in relation to a non-communicable disease (physical or mental health); and results were drawn from that part of the study only. CPGs produced for a health system, a group of healthcare professionals, a country, state, or province were included. Included studies described and assessed the guideline implementation strategy adopted. No restrictions were placed upon the type or number of strategies used. Studies that did not describe a guideline implementation strategy were excluded.

\section{Comparisons}

For studies targeting physical health conditions, included studies could compare a group where one or more CPG implementation strategies was used to a control group where no specific implementation strategy was adopted, or groups using different implementation strategies could be directly compared.

For studies targeting mental health conditions, included studies did not have to include a control group. In such cases, the comparison of interest was outcomes before and after the introduction of the CPG and supporting implementation strategy. For study designs employing control groups, included studies could compare a group where one or more CPG implementation strategies was used to a control group where no specific implementation strategy was adopted, or groups using different implementation strategies could be directly compared.

\section{Outcomes}

Included studies demonstrated pre and post measurement of processes or outcomes targeted by the CPG. Outcome measures could therefore concern the effectiveness of the implementation strategy measured by compliance with the CPG (for example prescribing behaviours), changes in the knowledge, attitudes, beliefs of behaviours of healthcare professionals, or changes in patients' health. Outcomes could be clinician or patient reported. Post-implementation measurement could take place at any point following the introduction of the CPG and accompanying implementation strategies. Studies that did not include pre and post CPG implementation measurement were excluded.

\section{Screening}

Screening of titles and abstracts and full-texts was conducted by four authors (MD, KS, HP and EE). Authors met to agree on final inclusion. Uncertainties experienced during the screening and extraction process were resolved with input from an additional author (LG).

\section{Data extraction and quality assessment}

Three authors (MD, KS and FA) independently extracted data from included studies and appraised the potential risk of bias in each study using tools developed by the Cochrane Effective Practice and Organisation of Care (EPOC) group [35]. An additional author (LG) double checked all data extractions and risk of bias assessments. The Cochrane Collaboration's 'EPOC taxonomy' was used to classify the implementation strategies adopted in included papers [36].

\section{Data synthesis}

Due to the nature of the review question there was heterogeneity in the participants, interventions, comparisons, outcome measures and outcomes of included studies. A descriptive data synthesis was consequently undertaken to summarise the characteristics and results from included studies in table form and to address the review questions.

\section{Results \\ Results of the search}

The electronic searches yielded 18,060 citations. 7950 remained after duplicates were removed. An additional 38 citations were uncovered by hand searching reference lists of key papers and through contact with experts in the field. This was reduced to 159 papers for full text screening, of which 6 met the inclusion criteria for the review. Details of included studies are provided in Table 1 and Fig. 2.

\section{Included studies}

Six papers met the inclusion criteria for the review. Four of these papers related to the introduction of CPGs for non-communicable diseases in physical health [37-40], 


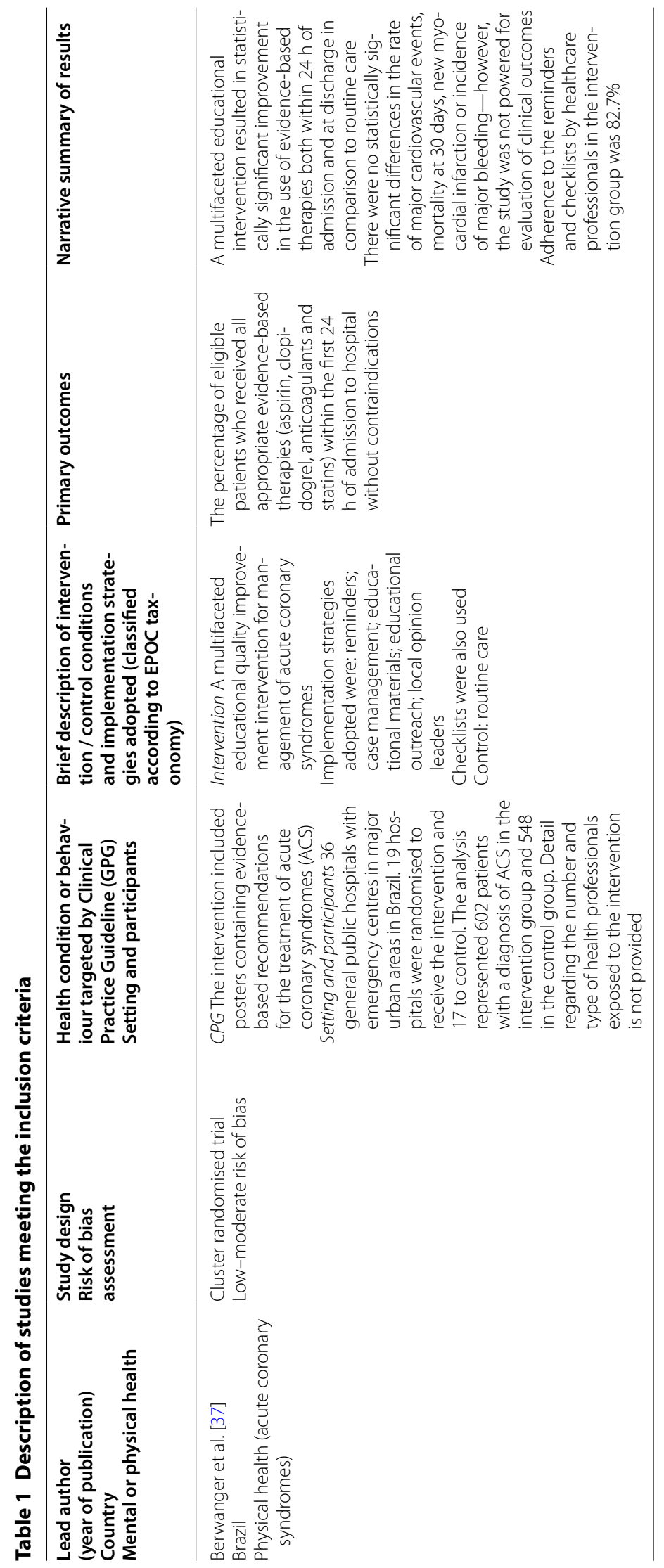




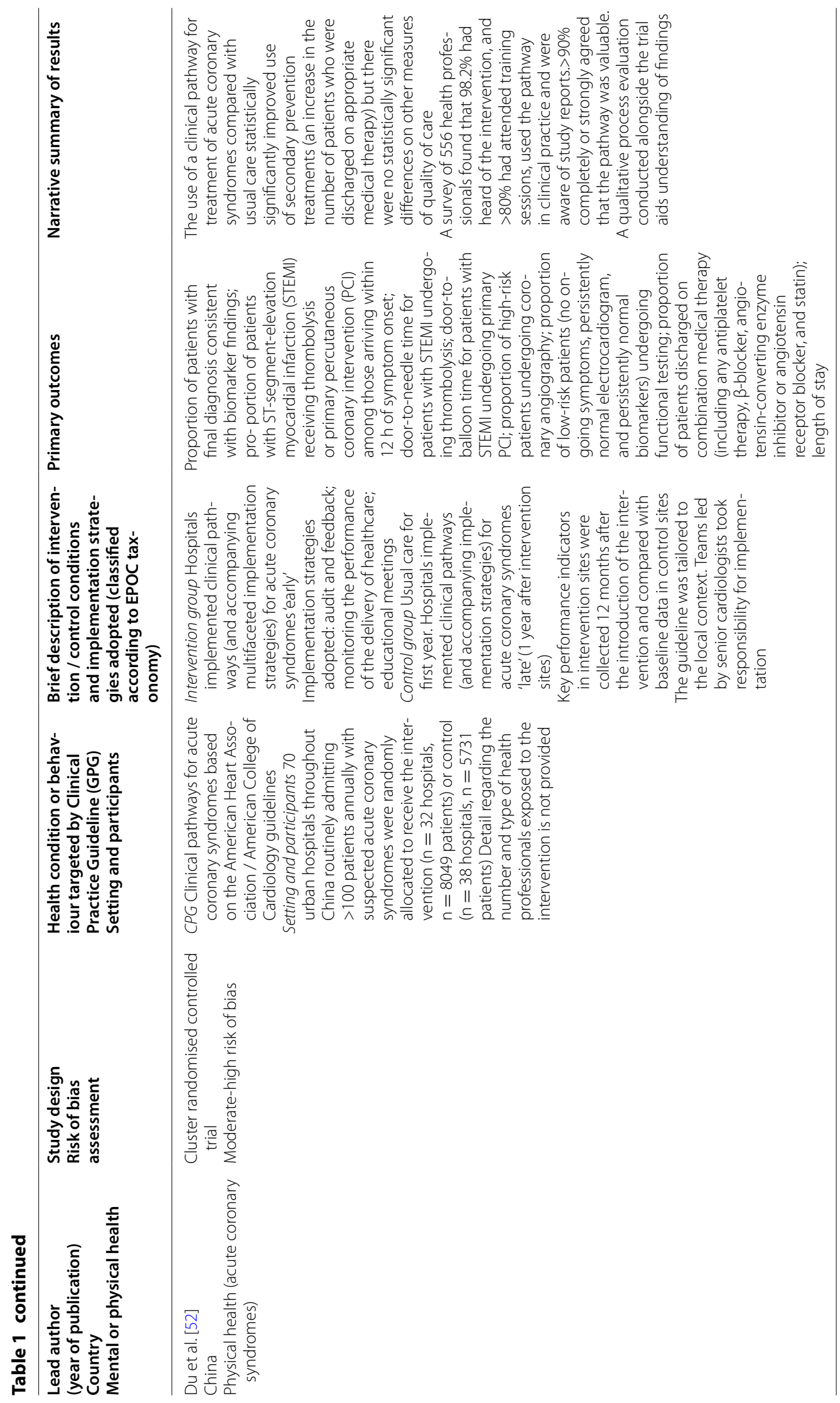




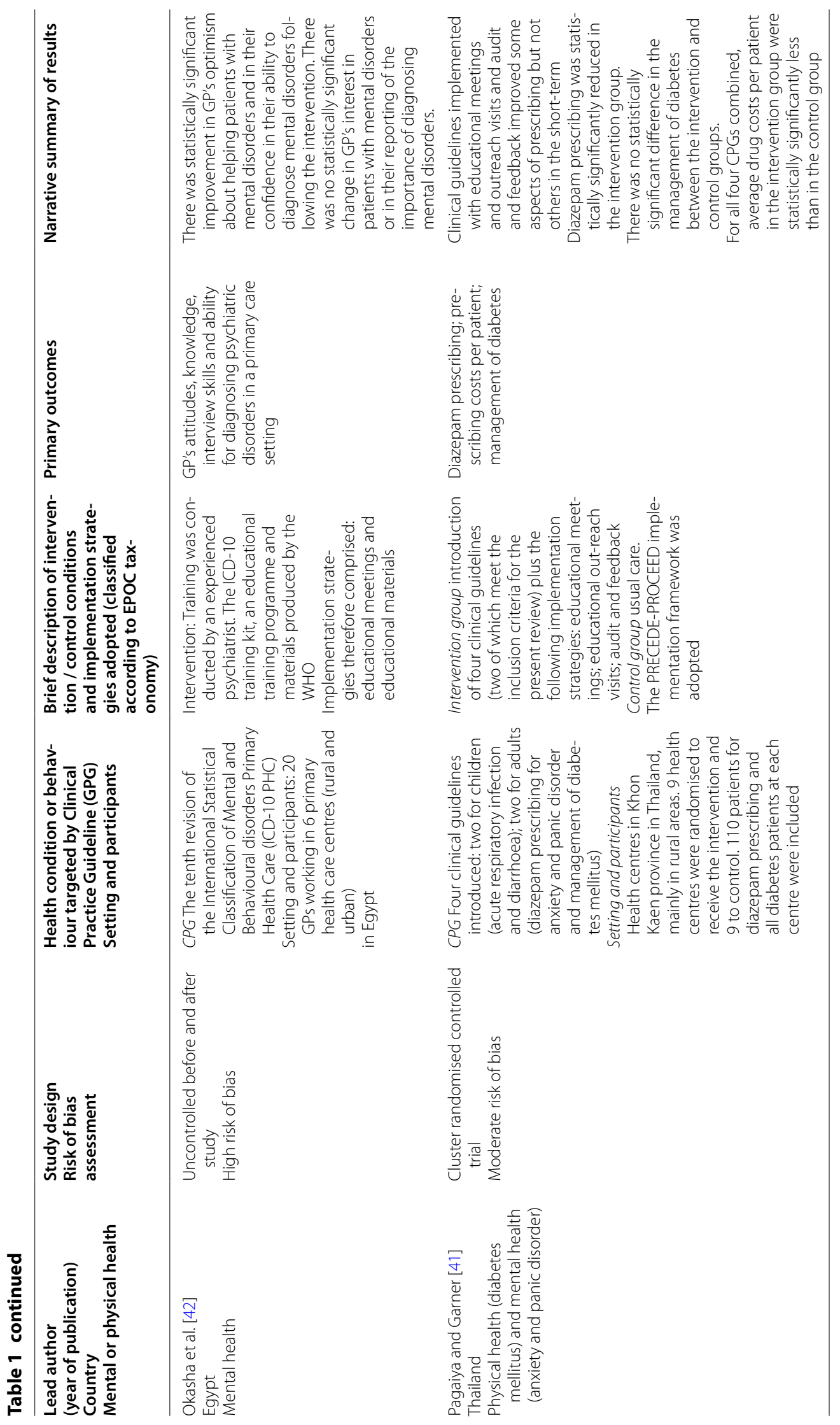




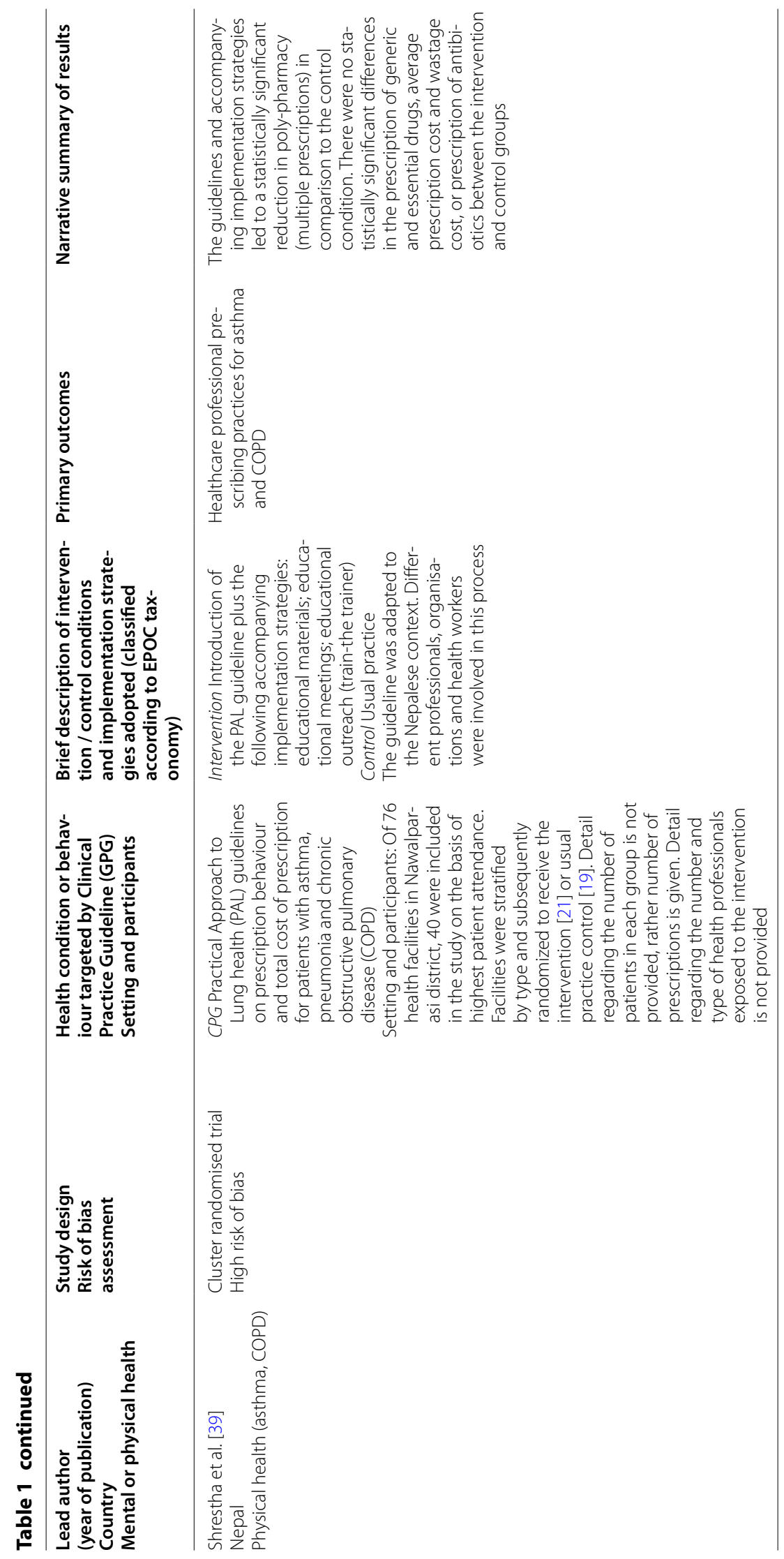




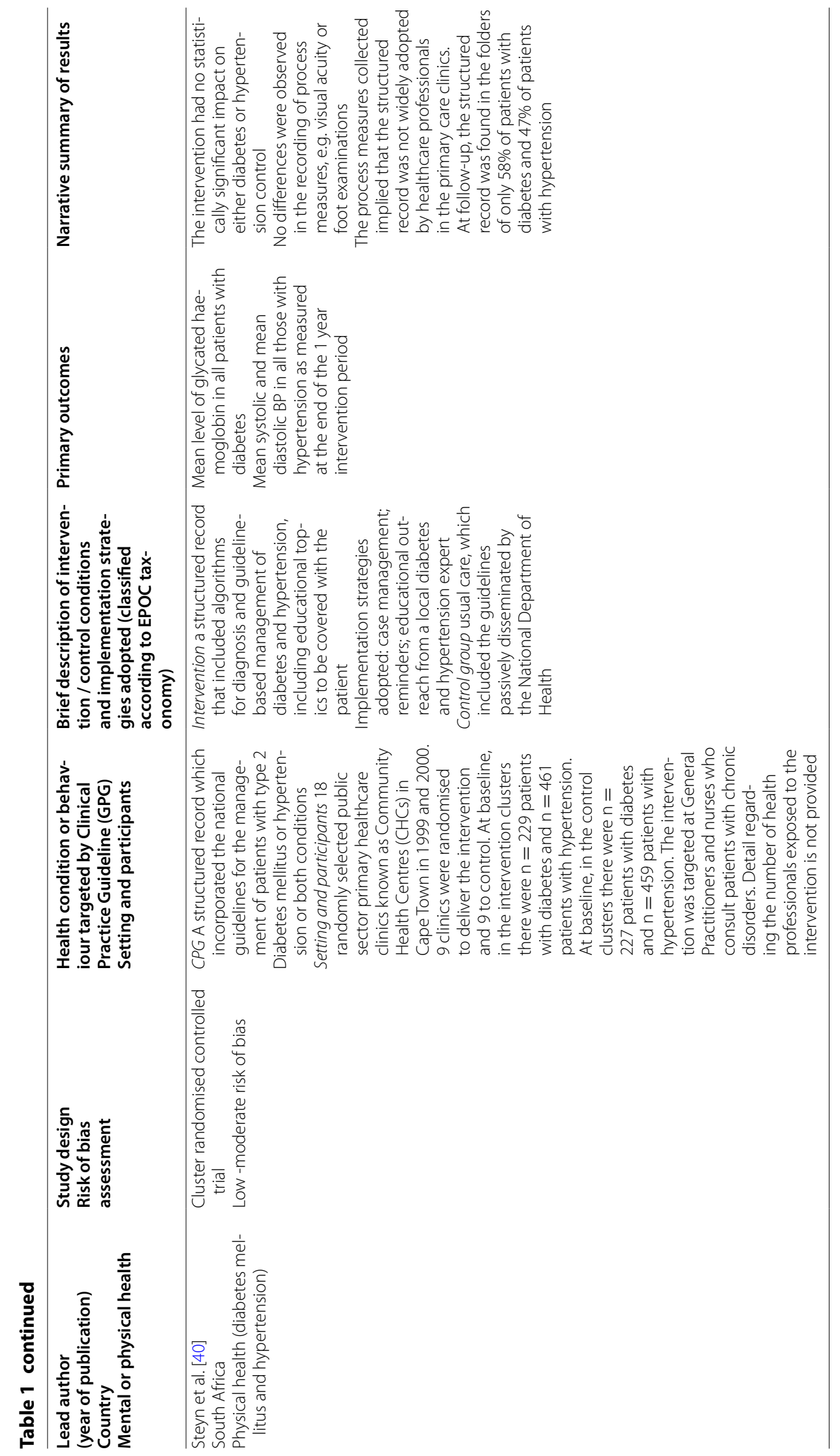




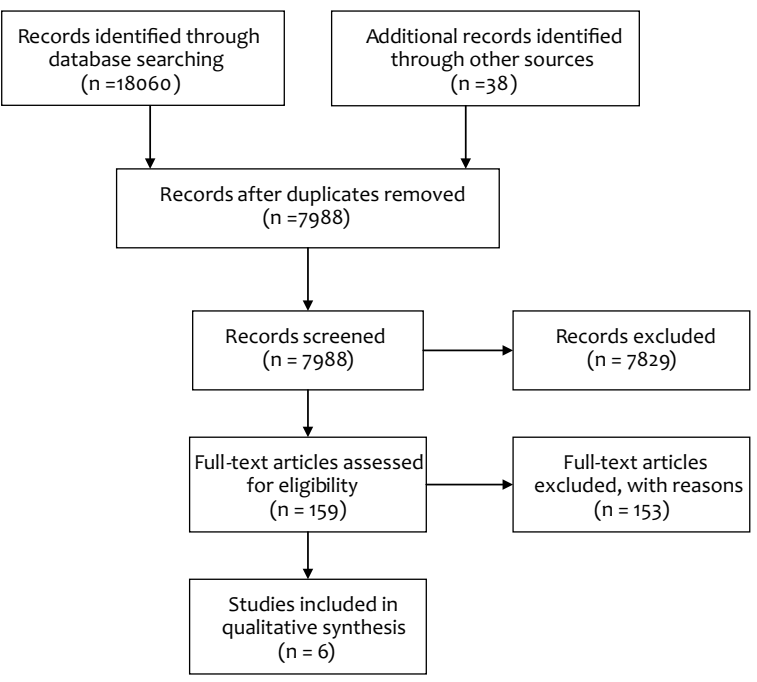

Fig. 2 PRISMA flow diagram of inclusion and exclusion process [33]

one paper included CPGs for both a non-communicable disease in physical health and mental health [41] and one paper examined the introduction of a CPG for mental health [42].

\section{Design}

Five cluster randomised controlled trials (cluster RCTs) [37-41] and one uncontrolled before and after study (in mental health) [42] were included in the review.

\section{Population}

Due to the nature of the interventions being reviewed (implementation of CPGs); all interventions were delivered to healthcare professionals. Where specified, participants receiving the intervention were typically physicians and nurses.

\section{Setting}

Included studies were conducted in Brazil [37], China [38], Thailand [41], Nepal [39], South Africa [40] and Egypt [42]. Two studies were set in general hospitals in urban areas [37, 38]. Four studies were set in primary health care centres: one of these was conducted in an urban area [40]; two in rural areas [39, 41] and one in a mix of urban and rural areas [42].

\section{Behaviour/clinical condition targeted by the CPG}

The non-communicable clinical conditions and behaviours targeted by the CPGs were as follows: use of evidence-based therapies for acute coronary syndromes [37, 38]; diazepam prescribing practices for anxiety or panic disorder and management of diabetes mellitus [41]; prescribing practices for asthma and COPD [39]; management of diabetes mellitus and hypertension [40]; and management of mental and behavioural disorders in primary healthcare through implementation of the ICD10 [42].

\section{Format of clinical practice guidelines}

Evidence-based guidelines were presented by: poster [37]; a clinical pathway document containing structured algorithms to be filled in by the healthcare professional [38]; a structured record to be filled in by the healthcare professional which was a three sided, folded A3 sheet of coloured paper placed with each patient's notes [40]; and A4 laminated documents printed on coloured paper [41]. In two studies, the format of the CPG was unclear [39, 42]. Four studies report that the CPG was either originally developed for the local context or tailored to the local context [38-41].

\section{CPG implementation strategies adopted}

All of the included studies adopted multi-faceted CPG implementation strategies. The components of the multifaceted interventions were sometimes poorly described. All studies employed implementation strategies that targeted healthcare professionals' use of the CPG. Implementation strategies adopted to facilitate uptake of CPGs (classified according to the EPOC taxonomy) were: reminders, case management, educational materials and educational outreach [37]; audit and feedback, monitoring the performance of the delivery of healthcare and educational meetings [38]; educational meetings, educational out-reach and audit and feedback [41]; educational materials, educational meetings and educational outreach [39]; case management, reminders and educational outreach [40]; educational meetings and educational materials [42].

Only one study [41] explicitly described adoption of a theoretical framework to guide CPG implementation. One paper reported the lack of adaptation of the CPG implementation strategies to the local context as a limitation [40] - this finding was pronounced in qualitative interviews undertaken with healthcare professionals as part of a process evaluation.

\section{Control groups}

In four of the cluster-RCTs, the intervention (CPG and accompanying implementation strategies) was compared to routine care (no CPG or accompanying implementation strategies) [37-39, 41]. In one cluster-RCT, the intervention (CPG and accompanying implementation strategies) was compared to passive diffusion of the CPG (no accompanying implementation strategies) [40]. The before and after study in mental health [42] did not include a control group. 


\section{Primary outcomes}

Primary outcomes relating to processes undertaken by healthcare professionals were: the proportion of patients who received evidence-based treatments for acute coronary syndromes $[37,38]$ and prescribing practices [39, 41]. Primary outcomes relating to healthcare professionals were: attitudes, knowledge and skills [42]. Primary outcomes relating to patients were level of glycated haemoglobin in patients with diabetes and systolic and diastolic blood pressure in patients with hypertension [40].

\section{Risk of bias in included studies}

The risk of bias in included studies was mixed. In the case of the cluster-RCTs, the nature of the intervention (CPG) and accompanying implementation strategies (e.g. educational sessions) often meant that it was not feasible to blind participants or personnel to the allocation of each cluster. In some cases, staff from different groups met occasionally leading to opportunity for contamination. Randomisation processes were judged to be low risk in four studies [37, 38, 40, 41] and unclear in one study [39]. Risk of bias in the mental health paper that used the ICD10 [42] was judged to be very high due to the use of an uncontrolled before and after study design.

\section{Effects of interventions}

Results of the included studies generally show statistically significant improvement on some but not all outcomes with the exception of one study [40] where the CPG intervention did not demonstrate any statistically significant improvement in comparison to control. The authors of this paper proposed that poor implementation of their intervention and accompanying implementation strategies was the major contributor to ineffectiveness.

Multifaceted implementation strategies were generally deemed to be effective in encouraging use of guidelines and creating positive change in outcomes. However, it is not possible to infer which approach to guideline implementation is most effective. Furthermore, the small number of included studies means that there are no observable trends according to clinical setting or topic addressed by the CPG (e.g. physical versus mental health).

\section{Implementation outcomes}

Implementation outcomes, as defined by Proctor et al. [43], were infrequently studied. Cost effectiveness of the interventions (e.g. taking into account the cost of delivering the CPG implementation strategies) was not assessed in any of the included studies. However, the effect of the intervention on prescription costs was calculated in two studies [39, 41]. Fidelity and acceptability of the intervention was explored via qualitative process evaluation in two studies $[38,40]$. The length of time to follow-up in included studies was generally short: 30 days [37]; 6 months [41]; 8 months [39]; 1 year [38, 40] and unclear but assumed to be immediately following the intervention [42]. This limits the ability to draw inferences regarding sustainability of any improved practice.

\section{Discussion}

The aims of this review were to examine studies of the effectiveness of evidence-based clinical practice guideline implementation for non-communicable diseases in LMICs, and to learn transferable lessons from other non-communicable diseases to mental health. The review revealed a significant paucity of good quality controlled studies not only in the field of mental health but across other non-communicable diseases in LMICs.

The small number of included papers made it difficult to draw clear conclusions about the role of different implementation strategies to support CPGs in mental health or in other non-communicable disease areas. Our inclusion criteria for mental health studies permitted uncontrolled designs in order to capture any existing work in this area. For other non-communicable disease areas, only high quality studies including control groups were included to support better validity of any conclusions being drawn about transferability of lessons learned across health sectors. As a result of this design, the relatively stringent inclusion criteria led to a large number of non-controlled studies in physical health being excluded from review. Future reviews may therefore wish to relax the inclusions criteria to gather a wider range of information which can create a conceptual map of this type of evidence.

This larger volume of excluded non-controlled studies in physical health had significant methodological limitations in addition to the absence of a control group. A number of studies described interventions with adequate study design for inclusion, but did not detail or refer to an evidence-base in the development of the CPG (e.g. [44]). Other common omissions included failure to clearly describe the implementation strategy used, to consider reproducibility of results, or to consider a theoretical framework in which to seat the implementation study and evaluation. Many excluded papers relied on pre and post analysis without clear attention to confounders that may have affected whether the implementation strategy itself, or other variables, impacted upon any observed change in practice. This difficulty is noted ten years after this problem had already been highlighted in a previous literature review [45]. Overall, the methodological limitations of the excluded studies (see Additional file 1: Web Appendix) mirrored similar problems reported within current literature on guideline implementation in 
high-income countries suggesting that these study design difficulties are consistent across these different health care settings [24, 27, 47].

Most of the included studies did not explore the relative efficacy of one implementation strategy compared to another in supporting the uptake of and adherence to CPGs, leaving important knowledge gaps with regards to the most effective and efficient strategies to support change within low resource settings. Additionally, there was a frequent lack of delineation between the effect of a strategy to improve uptake of guidelines distinct from whether improved implementation actually improved clinical outcomes targeted by the CPG (e.g. [40, 46]).

Multifaceted interventions with an educational component appear to be effective at supporting change, and attention to their delivery alongside CPG implementation is important to ensure optimum impact. Development of the CPG with consideration of local context, including staff attitudes and available resources, appear to be very important. The one study that showed no improvement in any targeted outcomes had developed CPGs specifically for the local context but had not sufficiently developed a feasible implementation strategy. Qualitative interviews with the professionals involved in implementing CPGs in this study revealed not only an ambivalence towards the perceived utility of the intervention, but also a fundamental failure of the guideline to accommodate resource limitations meaning that were the guideline to be fully implemented it would simply not be affordable [40]. In comparison, the studies that tailored both CPG development and implementation to the local context appeared to be relatively more efficacious in respect of achieving targeted outcomes [38-40].

Known barriers to improving mental health provision in LMICS includes the perceived lack of importance of this field relative to other clinical areas. These observations together support the impression that guideline implementers need to engage those using or impacted upon by the CPGS prior to implementing other strategies focused directly on the guideline use. In mental health this could for example include preliminary anti-stigma interventions. Pagaiya recommends that a "well-planned stepwise process be adopted which takes account of both theoretical and empirical evidence, as well as obstacles to change in relation to the individual staff and the local context" in order to approach successful implementation [41].

Prescribing featured in several of the included studies including one of those addressing mental health guidelines (the ICD-10), which may reflect the relatively more straightforward task of monitoring this sort of intervention. It could be argued that other mental health interventions such as the delivery of psychological therapy or case management approach are more challenging to formulate into guidelines and associated guideline adherence monitoring. Medication-based interventions in mental health CPGs implemented in high income countries may be a feasible place for future researchers to gain insight. Consideration of local availability of specific medications, facilities to store them and resource constraints on formulations offered will be essential in developing and implementing such guidelines in lower income settings.

There was an insufficient number of studies to be able to draw conclusions about transferability of findings to specific diseases, populations or care settings. However, the diversity of the included studies illustrates that CPGs can be implemented and evaluated across a range of populations and care settings within LMICs. Asthma, COPD, hypertension, acute coronary syndrome (ACS) and diabetes were targeted in the included studies on physical health conditions and primary care mental health provision and anxiety disorders targeted in the mental health paper. The transferability of lessons from most of the included physical illnesses to mental health seems feasible in principle due to the parallels between these disease courses and treatment requirements. Most conditions studied were chronic diseases which present with a risk of fluctuations and requirement of a series of interventions to support recovery and stability, in keeping with the clinical course of many mental health disorders (e.g. schizophrenia). ACS is an acute presentation with the requirement of treatment intervention and systematic follow up for a period in keeping with most mental health crises (e.g. suicide plans). There were insufficient studies to explore the question of whether similar pathways and protocols can be constructed for mental health conditions but the findings did not exclude the transferability of these approaches.

Despite these similarities in principle supporting the transferability of implementation experience across mental health and other non-communicable disease areas, there are also important differences in these fields. Mental health workers employ different diagnostic processes, with less reliance on technology and more reliance on human resources to deliver both assessment and treatment. These features highlight a risk for even greater variation in practices and the need to learn from experience in other non-communicable disease areas of the importance of embedding systems for standardisation and measurement of interventions within guidelines. Outcomes in mental health are frequently qualitatively different to those in other non-communicable diseases and less amenable to conventional measurements. In the absence of biomarker outcome measures the importance of developing and incorporating simple and tractable 
measures such as quality of life, ability to sustain employment, activities of daily living (ADLs) or relationships in order to validate and monitor the efficacy of CPGs in this field is essential.

Local context including prevalent knowledge, behaviours and attitudes towards mental health conditions has predicted larger impact on the potential success of a guideline than in many of the non-communicable diseases considered in this review. Despite some literature acknowledging the context in which a guideline was to be implemented, few gave consideration to the range of barriers that would need to be considered in mental health before implementing and evaluating change. Although beyond the scope of this paper, an important area to consider in future reviews is how impediments to changes in practice due to stigma in some communicable disease areas, for example HIV, have been overcome and addressed in guideline development and implementation.

A consistent difficulty observed in included studies was the limited follow up to evaluate longer-term impact and interventions needed to embed changes in clinical practice in the medium and long term. Longer follow up periods are needed to understand the requirements for sustained change such as on-going interventions (e.g. educational updates) or wider system changes (e.g. changes in job roles, informatics or care pathways and checklists). There is evidence that active on-going efforts to support changes in practice may be required beyond the initial implementation period (e.g. [45, 48, 49]. This has important resource implications for health planners looking to embed improvements into routine clinical care. None of the included studies conducted cost effectiveness analyses of CPG implementation which is a very important omission given the resource constraints in these settings. This should be considered a clear priority in future study designs.

Despite most of the included studies showing improvements in a selection of outcomes, it is not possible to reach conclusions regarding the sustainability, feasibility or practicality of these approaches for mental health planners. For example, all included studies used educational implementation strategies and showed them to be broadly efficacious but they can be labour intensive. One difficulty in providing such strategies in LMICs is the available modes and associated resources for delivering the educational intervention. Furthermore, education is an important tool to support changes in clinical practice but rarely a one off solution to altering knowledge and behaviours. Multiple sessions and top ups are usually required alongside rolling programmes to support staff turnover. Implementing interventions based around education usually require additional strategies such as rigorous systems for monitoring to support change. Outreach approaches were used in some of the studies but again these can be costly and if practitioners are geographically very far apart, this would make educational meetings difficult. Educational sessions also reduce time to engage in clinical activity in already resource stretched services, this places an onus on organisers to ensure high quality sessions or material to support perceived utility and buy in towards the CPG.

Another implementation strategy commonly used in low resource settings, known as task shifting, was not adopted in the studies included within this review. Task shifting refers to the process of transferring a task usually delivered by a scare resource such as a physician to a more rapidly trained and less scare resource such as a health care worker. Evidence has shown that this strategy has been effective in increasing use of treatment guides and protocols in the management of a range of noncommunicable conditions such as asthma, hypertension, epilepsy, diabetes and depression [50, 51]. Integrated protocols that involved strategies such as care bundles or care pathways that supported whole systems change seem potentially more promising than focus on just the guideline alone (e.g. [52]). Directed studies to explore the use of task shifting alongside other CPG implementation strategies may be an important area for future research in LMICs considering the need for CPGs not only to improve patient outcomes but also to address efficiency.

Despite the emergence of some lessons for mental health planners, the volume of literature excluded from this review (see Additional file 1: Web Appendix) reveals significant limitations in both study methodology and reporting practices and a need to increase the volume of good quality research. This highlights the importance of a concerted effort within LMICs to improve the rigor of CPG implementation studies. Efforts to increase the availability and existence of context adapted evidencebased mental health guidelines are in process but it is essential that mental health care planners and researchers learn from the limitations encountered in other non-communicable disease guideline implementation research. The World Health Organization mhGAP Intervention Guide, for example, is now in use in over 90 countries worldwide, where the guidelines are intended to be locally adapted for each country and each context, but as yet few evaluations of its use have been published [29, 30, 53-55].

This raises the more fundamental question of whether the scope of this review was too narrow. Clearly this field is not at the stage where many RCTs have been published, from which strong summary findings can be draw. Indeed, given the relative infancy of this field, we did consider whether to conduct a broader narrative review of the literature, for example to summarise what is known 
of barrier and facilitator factors in guideline implementation in LMICs. Similarly we considered including process evaluation papers in this review. On balance we decided to take a narrower focus on the better quality papers for this paper. The results indicate that future reviews may need to adopt somewhat broader criteria, for example including non-experimental studies, until such time as the quality of the available evidence improves sufficiently.

The overall disconnection which was observed between the volume of literature on guideline development in LMICs, and that on guideline implementation raises important questions for those developing and adapting guidelines in mental health about with whom the responsibility lies for ensuring validation and implementation studies of CPGs are conducted. It has been argued that sound validation studies should be considered a prerequisite for conferring the label of an evidence-based mental health guideline [26]. The importance of this approach in the field of mental health in lower resource settings has particular resonance due to the highly variable existing health care resources and infrastructures in which mental health care can be delivered and the requirement of a local approach to map knowledge, attitudes and behaviour before developing an implementation strategy. In resource limited settings with such significant diagnosis and treatment gaps, efforts to increase effective coverage of mental health care must consider implementation strategies and attention to resource constraints as necessary components of the guideline development process.

This review demonstrates the salience of the implementation gap across medical specialities in LMICs and the real risks for mental health in not responding to these lessons from CPG development over the last few decades. Those looking to scale up mental health in LMICs must prioritise implementation research. Those facilitating the development of CPGs, including professional bodies contributing to them, have a responsibility to ensure that their efforts and the money invested in them lead to tangible improvements in care. Unless agreement is made as to how to take forward this requirement for mental health provision, a real opportunity to learn from lessons in other sectors and settings will be missed.

\section{Conclusions}

Current evidence for the effectiveness of interventions to improve uptake of and compliance with evidence based guidelines in LMICs for mental disorders and for other non-communicable diseases is very limited. The literature suggests that multifaceted CPG implementation strategies that involve an educational component may be an effective way of improving guideline adherence and therefore improving clinical outcomes. Further work is needed to examine cost effectiveness of CPG implementation strategies in LMICs and to draw conclusions on the transferability of implementation experience in other non-communicable disease areas to mental health. Strategies to ensure that CPGs are developed with clear guidance for implementation and methods to evaluate them should be a priority for mental health researchers and for international agencies.

\section{Additional file}

Additional file 1: Web Appendix. Excluded papers.

\section{Abbreviations}

ACS: acute coronary syndrome; COPD: chronic obstructive pulmonary disorder; CPGs: clinical practice guidelines; EPOC: Cochrane Effective Practice and Organisation of Care; CBA: controlled-before-and-after studies; CCT: controlled clinical trials; GNI: gross national income; ITS: interrupted time series; LMICs: Low and Middle Income Countries; PRISMA: preferred reporting items for systematic reviews and meta-analyses; RCTs: randomised controlled trials.

\section{Authors' contributions}

MD helped to develop the design of the review, conducted screening and data extraction, contributed to exclusion and exclusion criteria, drafted the discussion section of the manuscript and co-ordinated production of the manuscript. KS conducted screening and data extraction. LG helped to develop the design of the review, checked inclusions and data extraction, and drafted the methods and results sections of the manuscript. HP helped to develop the design of the review, wrote the search strategy, conducted the search, and conducted screening. EE conducted screening and reviewed inclusion and exclusion criteria. FA conducted data extraction. GT conceived the review, helped to develop the design of the review, and drafted the introduction. All authors provided comments on drafts of the manuscript and approved the final version to be published. All authors read and approved the final manuscript.

\section{Author details}

${ }^{1}$ South London and Maudsley NHS Foundation Trust, The Maudsley Hospital, Denmark Hill, London SE5 8AF, UK. ${ }^{2}$ Centre for Implementation Science, Institute of Psychiatry, Psychology and Neuroscience, King's College London, London, UK. ${ }^{3}$ Centre for Global Mental Health and Centre for Implementation Science, Institute of Psychiatry, Psychology and Neuroscience, King's College London, London, UK.

\section{Acknowledgements}

GT is supported by the National Institute for Health Research (NIHR) Collaboration for Leadership in Applied Health Research and Care South London at King's College London Foundation Trust. The views expressed are those of the author(s) and not necessarily those of the NHS, the NIHR or the Department of Health. GT acknowledges financial support from the Department of Health via the National Institute for Health Research (NIHR) Biomedical Research Centre and Dementia Unit awarded to South London and Maudsley NHS Foundation Trust in partnership with King's College London and King's College Hospital NHS Foundation Trust. GT is supported by the European Union Seventh Framework Programme (FP7/2007-2013) Emerald project.

\section{Competing interests}

The authors declare that they have no competing interests.

Availability of data and supporting materials

There is no additional data associated with this work. A Additional file of the review search strategy used has been submitted.

\section{Consent for publication}

Not applicable. This is a review article and does not contain any individual person's data. 


\section{Ethics approval and consent to participate}

Not applicable. This is a review article and does not report on or involve the use of any animal or human data or tissue.

\section{Funding}

KS, LG, HP and EE were supported by King's Improvement Science. King's Improvement Science is part of the NIHR CLAHRC South London and comprises a specialist team of improvement scientists and senior researchers based at King's College London. It's work is funded by King's Health Partners (Guy's and St Thomas' NHS Foundation Trust, King's College Hospital NHS Foundation Trust, King's College London and South London and Maudsley NHS Foundation Trust), Guy's and St Thomas' Charity, the Maudsley Charity and the Health Foundation. There was no direct individual funding for this work. MD did not receive funding for this work. There was no distinct role of any funding body in the design of the study and collection, analysis, and interpretation of data and in writing the manuscript as detailed in contributions.

Received: 8 November 2016 Accepted: 21 December 2016 Published online: 05 January 2017

\section{References}

1. Fekadu A, Medhin G, Kebede D, Alem A, Cleare A, Prince M, et al. Excess mortality in severe mental disorders: a 10-year population-based cohort study in rural Ethiopia. Br J Psychiatry. 2015;206(4):289-96. doi:10.1192/ bjp.bp.114.149112.

2. Whiteford HA, Degenhardt L, Rehm J, Baxter AJ, Ferrari AJ, Erskine HE, et al. Global burden of disease attributable to mental and substance use disorders: findings from the Global Burden of Disease Study 2010. Lancet. 2013;382(9904):1575-86.

3. Prince M, Patel V, Saxena S, Maj M, Maselko J, Phillips MR, et al. Global mental health 1: no health without mental health. Lancet. 2007:370:859-77.

4. Drew N, Funk M, Tang S, Lamichhane J, Chávez E, Katontoka S, et al. Human rights violations of people with mental and psychosocial disabilities: an unresolved global crisis. Lancet. 2011;378(9803):1664-75.

5. Alem A, Kebede D, Fekadu A, Shibre T, Fekadu D, Beyero T, et al. Clinical course and outcome of schizophrenia in a predominantly treatmentnaive cohort in rural Ethiopia. Schizophr Bull. 2009;35(3):646-54.

6. Wang PS, Aguilar-Gaxiola S, Alonso J, Angermeyer MC, Borges G, Bromet EJ, et al. Use of mental health services for anxiety, mood, and substance disorders in 17 countries in the WHO world mental health surveys. Lancet. 2007:370(9590):841-50.

7. Thornicroft G. Most people with mental illness are not treated. Lancet. 2007;370(9590):807-8.

8. Vigo D, Thornicroft G, Atun R. Estimating the true global burden of mental illness. Lancet Psychiatry. 2016;3(2):171-8.

9. Peters $\mathrm{DH}$, Adam T, Alonge O, Agyepong IA, Tran N. Implementation research: what it is and how to do it. BMJ. 2013;347:f6753.

10. Thornicroft G. Evidence-based mental health care and implementation science in low- and middle-income countries. Epidemiol Psychiatr Sci. 2012;21(3):241-4.

11. Jacob KS, Sharan P, Mirza I, Garrido-Cumbrera M, Seedat S, Mari JJ, et al. Mental health systems in countries: where are we now? Lancet. 2007:370(9592):1061-77.

12. Draper CE, Lund C, Kleintjes S, Funk M, Omar M, Flisher AJ. Mental health policy in South Africa: development process and content. Health Policy Plan. 2009;24(5):342-56

13. Bird P, Omar M, Doku V, Lund C, Nsereko JR, Mwanza J. Increasing the priority of mental health in Africa: findings from qualitative research in Ghana, South Africa, Uganda and Zambia. Health Policy Plan 2010.

14. Saraceno B, Van OM, Batniji R, Cohen A, Gureje O, Mahoney J, et al. Barriers to improvement of mental health services in low-income and middleincome countries. Lancet. 2007:370(9593):1164-74.

15. Petersen I, Lund C, Stein DJ. Optimizing mental health services in low-income and middle-income countries. Curr Opin Psychiatry. 2011;24(4):318-23.
16. Saraceno B, van Ommeren M, Batniji R, Cohen A, Gureje O, Mahoney J, et al. Barriers to improvement of mental health services in low-income and middle-income countries. Lancet. 2007;370(9593):1164-74.

17. Swanson RC, Cattaneo A, Bradley E, Chunharas S, Atun R, Abbas KM, et al. Rethinking health systems strengthening: key systems thinking tools and strategies for transformational change. Health Policy Plan. 2012;27(Suppl 4):iv54-61.

18. WHO. Health systems: improving performance. Geneva: World Health Organization;2000.

19. Da Silva MCA, Patel V. Evaluations of effectiveness in the real world. In: Thornicroft GPV, editor. Global mental health trials. Oxford: Oxford University Press; 2014

20. Hanlon C, Fekadu A, Jordans M, Kigozi F, Petersen I, Shidhaye R, et al. District mental healthcare plans for five low- and middle-income countries: commonalities, variations and evidence gaps. Br J Psychiatry. 2015.

21. Rowe LA, Brillant SB, Cleveland E, Dahn BT, Ramanadhan S, Podesta M, et al. Building capacity in health facility management: guiding principles for skills transfer in Liberia. Hum Resour Health. 2010;8:5

22. World Health Organization. Mental health systems in selected low- and middle-income countries: a WHO-AIMS cross-national analysis: WHO; 2009.

23. Curry LA, Alpern R, Webster TR, Byam P, Zerihun A, Tarakeshwar N, et al. Community perspectives on roles and responsibilities for strengthening primary health care in rural Ethiopia. Glob Public Health. 2012;7(9):961-73.

24. Grimshaw J, Eccles M, Thomas R, MacLennan G, Ramsay C, Fraser C, et al. Toward evidence-based quality improvement. Evidence (and its limitations) of the effectiveness of guideline dissemination and implementation strategies 1966-1998. J Gen Intern Med. 2006:21(Suppl 2):S14-20.

25. Bauer MS. A review of quantitative studies of adherence to mental health clinical practice guidelines. Harv Rev Psychiatry. 2002;10(3):138-53.

26. Weinmann S, Koesters $M$, Becker T. Effects of implementation of psychiatric guidelines on provider performance and patient outcome: systematic review. Acta Psychiatr Scand. 2007;115(6):420-33.

27. Barbui C, Girlanda F, Ay E, Cipriani A, Becker T, Koesters M. Implementation of treatment guidelines for specialist mental health care. Cochrane Database Syst Rev. 2014;1:CD009780.

28. Girlanda F, Fiedler I, Ay E, Barbui C, Koesters M. Guideline implementation strategies for specialist mental healthcare. Curr Opin Psychiatry. 2013:26(4):369-75.

29. Barbui C, Dua T, van Ommeren M, Yasamy MT, Fleischmann A, Clark N, et al. Challenges in developing evidence-based recommendations using the GRADE approach: the case of mental, neurological, and substance use disorders. Plos Med. 2010;7(8):e1000322

30. Dua T, Barbui C, Clark N, Fleischmann A, Poznyak V, van Ommeren M, et al. Evidence-based guidelines for mental, neurological, and substance use disorders in low- and middle-income countries: summary of WHO recommendations. Plos Med. 2011;8(11):e1001122.

31. World Health Organization. WHO mental health atlas. Geneva: World Health Organization; 2011.

32. HJPTG S, editor. Cochrane Handbook for Systematic Reviews of Interventions. 2011

33. Moher D, Liberati A, Tetzlaff J, Altman DG. Reprint-preferred reporting items for systematic reviews and meta-analyses: the PRISMA statement. Phys Ther. 2009;89(9):873-80.

34. Medicine lo. Clinical practice guidelines we can trust. Institute of Medicine; 2011.

35. EPOC. Effective practice and organisation of care (EPOC) resources for review authors. Oslo: Norwegian Knowledge Centre for the Health Services; 2015. http://epoc.cochrane.org/ epoc-specific-resources-review-authors.

36. EPOC. EPOC taxonomy. 2015. https://epoc.cochrane.org/epoc-taxonomy.

37. Berwanger O, Guimaraes HP, Laranjeira LN, Cavalcanti AB, Kodama AA, Zazula AD, et al. Effect of a multifaceted intervention on use of evidencebased therapies in patients with acute coronary syndromes in Brazil: the BRIDGE-ACS randomized trial. JAMA. 2012;307(19):2041-9.

38. Du X, Gao R, Turnbull F, Wu Y, Rong Y, Lo S, et al. Hospital quality improvement initiative for patients with acute coronary syndromes in China: a cluster randomized, controlled trial. Circ Cardiovasc Qual Outcomes. 2014;7(2):217-26. 
39. Shrestha N, Samir KC, Baltussen R, Kafle KK, Bishai D, Niessen L. Practical approach to lung health in Nepal: better prescribing and reduction of cost. Trop Med Int Health. 2006;1 1(5):765-72.

40. Steyn K, Lombard C, Gwebushe N, Fourie JM, Everett-Murphy K, Zwarenstein $\mathrm{M}$, et al. Implementation of national guidelines, incorporated within structured diabetes and hypertension records at primary level care in Cape Town, South Africa: a randomised controlled trial. Glob Health Action. 2013;6:20796.

41. Pagaiya N, Garner P. Primary care nurses using guidelines in Thailand: a randomized controlled trial. Trop Med Int Health. 2005;10(5):471-7.

42. Okasha AFM, Haggag $W$, et al. A psychiatric training programme for general practitioners in primary health care in Egypt. Prim Care Psychiatry. 2002;8(1):9-16.

43. Proctor E, Silmere H, Raghavan R, Hovmand P, Aarons G, Bunger A, et al. Outcomes for implementation research: conceptual distinctions, measurement challenges, and research agenda. Adm Policy Ment Health. 2011;38(2):65-76.

44. Deng Y, Liang L, Liu J, Jiang H. Analysis of monitoring results of iodine deficiency disorders in Ankang, Shaanxi province in 2012. Chin J Control Endemic Dis. 2013;28(4):284-6.

45. Siddiqi K, Newell J, Robinson M. Getting evidence into practice: what works in developing countries? Int I Qual Health Care. 2005;17(5):447-54.

46. Okasha A, Fahmy M, Haggag W, Awad M, Okasha T, Moez K. A psychiatric training programme for general practitioners in primary health care in Egypt. Primary Care Psychiatry. 2002;8(1):9-16.

47. Brusamento S, Legido-Quigley H, Panteli D, Turk E, Knai C, Saliba V, et al. Assessing the effectiveness of strategies to implement clinical guidelines for the management of chronic diseases at primary care level in EU Member States: a systematic review. Health Policy. 2012;107(2/3):168-83.
48. Rowe AK, Onikpo F, Lama M, Osterholt DM, Rowe SY, Deming MS. A multifaceted intervention to improve health worker adherence to integrated management of childhood illness guidelines in Benin. Am J Public Health. 2009;99(5):837-46.

49. Rowe AK, Osterholt DM, Kouame J, Piercefield E, Herman KM, Onikpo $F$, et al. Trends in health worker performance after implementing the integrated management of childhood illness strategy in Benin. Trop Med Int Health. 2012;17(4):438-46.

50. Joshi R, Alim M, Kengne AP, Jan S, Maulik PK, Peiris D, et al. Task shifting for non-communicable disease management in low and middle income countries—a systematic review. Plos ONE. 2014;9(8):e103754.

51. Petersen I, Lund C, Bhana A, Flisher AJ. A task shifting approach to primary mental health care for adults in South Africa: human resource requirements and costs for rural settings. Health Policy Plan. 2012;27(1):42-51.

52. Du X, Gao R, Turnbull F, Wu Y, Rong Y, Lo S, et al. Hospital quality improvement initiative for patients with acute coronary syndromes in China: a cluster randomized, controlled trial. Circ Cardiovasc Qual Outcomes. 2014;7(2):217-26.

53. World Health Organization. mhGAP intervention guide for mental, neurological and substance use disorders in non-specialized health settings: mental health Gap Action Programme (mhGAP). Geneva: WHO; 2010.

54. Siriwardhana C, Adikari A, Van Bortel T, McCrone P, Sumathipala A. An intervention to improve mental health care for conflict-affected forced migrants in low-resource primary care settings: a WHO MhGAP-based pilot study in Sri Lanka (COM-GAP study). Trials. 2013;14:423.

55. Abdulmalik J, Kola L, Fadahunsi W, Adebayo K, Yasamy MT, Musa E, et al. Country contextualization of the mental health gap action programme intervention guide: a case study from Nigeria. PLoS Med. 2013;10(8):e1001501.

\section{Submit your next manuscript to BioMed Central and we will help you at every step:}

- We accept pre-submission inquiries

- Our selector tool helps you to find the most relevant journal

- We provide round the clock customer support

- Convenient online submission

- Thorough peer review

- Inclusion in PubMed and all major indexing services

- Maximum visibility for your research

Submit your manuscript at www.biomedcentral.com/submit
() BioMed Central 